6. Marlhens F, Bareil C, Griffoin JM, et al. Mutations in RPE65 cause Leber's congenital amaurosis. Nat Genet 1997; 17: 139-41.

7. Gu SM, Thompson DA, Srikumari CR, et al. Mutations in RPE65 cause autosomal recessive childhoodonset severe retinal dystrophy. Nat Genet 1997; 17: 194-7.
8. Thompson DA, Li Y, McHenry CL, et al. Mutations in the gene encoding lecithin retinol acyltransferase are associated with early-onset severe retinal dystrophy. Nat Genet 2001; 28: 123-4.

9. Kuska V, Imanishi Y, Batten M, et al. Retinoid cycle in the vertebrate retina: experimental approaches and mechanisms of isomerization. Vision Res 2003 43: 2959-81.

10. Stockton DW, Lewis RA, Abboud $\varepsilon B$, et al. A novel locus for Leber congenital amaurosis on

chromosome 14q24. Hum Genet 1998; 103: 328-33.

\title{
NOUVELLE
}

\section{PCSK9 : un nouveau gène impliqué dans I'hypercholestérolémie familiale}

Gilles Lambert, Philippe Costet, Michel Krempf, Florent Lalanne
> L’hypercholestérolémie familiale se traduit cliniquement par des concentrations plasmatiques élevées de lipoprotéines de basse densité (LDL, low density lipoproteins), par des dépôts de cholestérol dans les tendons et la peau (xanthomes), ainsi que dans les artères (athérome), provoquant des accidents cardiovasculaires prématurés. Dans la plupart des cas recensés, c'est un désordre génétique à transmission autosomique dominante provenant de mutations dans le gène codant pour le récepteur des LDL qui est à l'origine de cette maladie [1]. Des mutations du gène codant pour l'apolipoprotéine $B 100$ (apoB100), notamment dans le domaine permettant la reconnaissance et la capture par le récepteur des LDL de l'apoB100, provoquent également un phénotype d'hypercholestérolémie familiale (familial ligand-defective apoB100) [1]. Enfin, dans de très rares familles où l'hypercholestérolémie familiale est à transmission autosomique récessive, la mutation du gène $A R H$ codant pour une protéine cytosolique du même nom, impliquée dans le processus d'incorporation du récepteur des LDL au niveau des puits de clathrine, permettant l'endocytose des LDL, est responsable du phénotype [1].

\section{Un troisième locus}

Récemment, deux équipes ont identifié un troisième locus dans la région du chromosome lp32 impliqué dans l'hypercholestérolémie familiale à transmission autosomique dominante $[2,3]$. À ce jour, quatre familles ont été identifiées: elles présentent des mutations (S127R, F216L, D374Y, N157K) sur un nouveau gène, PCSK9 (proprotein convertase subtilisin/kexin type 9) situé dans ce locus; ni le récepteur des LDL, ni l'apoB100 ne sont mutés [4-6]. PCSK9 est une proprotéine convertase, exprimée notamment dans le foie, l'épithélium intestinal, le rein et les neurones. PCSK9 serait impliquée dans la différenciation neuronale et pendant la régénération hépatique [7].

Le seul substrat connu de PCSK9 étant PCSK9 lui-même, son rôle physiologique est délicat à déterminer [7]. La première indication notable est venue de l'observation d'une régulation négative de PCSK9 par les stérols alimentaires et de la régulation positive de l'expression hépatique de PCSK9 chez des souris surexprimant SREBPla et SREBP2, deux facteurs de transcription activés lors d'une déplétion intracellulaire en cholestérol $[8,9]$. Ces observations ont été renfor- cées par la description d'une induction de l'expression de PCSK9 par les statines qui sont des inhibiteurs de I'HMG-CoA réductase, étape limitante de la biosynthèse du cholestérol [10].

\section{PCSK9 inhibe l'expression et l'activité} hépatique du récepteur des LDL

La surexpression de PCSK9 chez la souris (au moyen d'adénovirus recombinants) induit un doublement de la concentration de cholestérol circulant, en augmentant exclusivement le cholestérol des LDL [11]. Ce phénomène est associé à une diminution de l'expression hépatique du récepteur des LDL. En revanche, la surexpression de PCSK9, chez des souris dont le gène codant pour le récepteur des LDL a été invalidé, n'a pas d'effet sur les lipides plasmatiques. Ces résultats indiquent que PCSK9 inhibe l'expression du récepteur des LDL, ce qui induit une augmentation des concentrations plasmatiques de cholestérol LDL [11].

L'utilisation de modèles cellulaires (hépatomes HuH7 en culture) a permis de mettre en évidence un effet de PCSK9 sur la capture des LDL: la surexpression (transfection transitoire) ou l'atténuation (interférence par l'ARN) de PCSK9 provoquent respectivement une diminution et une augmentation de la capture cellulaire de LDL 
fluorescents (Figure 1). Par ailleurs, nous venons de montrer que la surexpression de PCSK9 chez la souris se traduit par un retard du catabolisme hépatique des LDL, similaire à ce qui est observé chez les souris déficientes en récepteur des LDL (G. Lambert, données non publiées). Les études de la mutation S127R de PCSK9 ont donné des résultats similaires à ceux des études de surexpression de PCSK9. En effet, des fibroblastes immor- talisés de patients présentant la mutation S127R de PCSK9 ont une diminution de l'expression du récepteur des LDL à leur surface par rapport à celle des fibroblastes témoins [12]. Chez deux patients ayant une mutation S127R, nous avons montré que le catabolisme des LDL est retardé de $30 \%$ chez ces patients par rapport aux témoins [13]. Cela suggère que certaines mutations se traduisent par un gain de fonction de PCSK9 plutôt
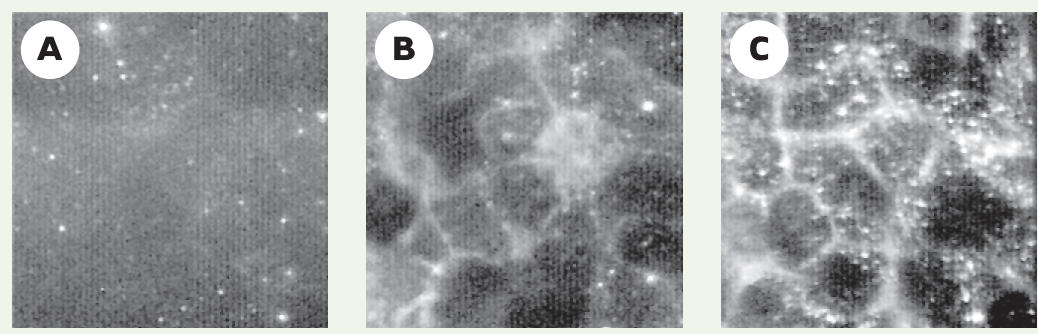

Figure 1. PCSK9 diminue la capture cellulaire des LDL. Capture de Dil-LDL fluorescents par des hépatomes humains (HuH7) présentant différentes valeurs d'expression de PCSK9. A. HuH7 surexprimant PCSK9 par transfection transitoire. B. HuH7 témoins. C. HuH7 dont l'expression endogène de PCSK9 est atténuée par interférence par l'ARN.

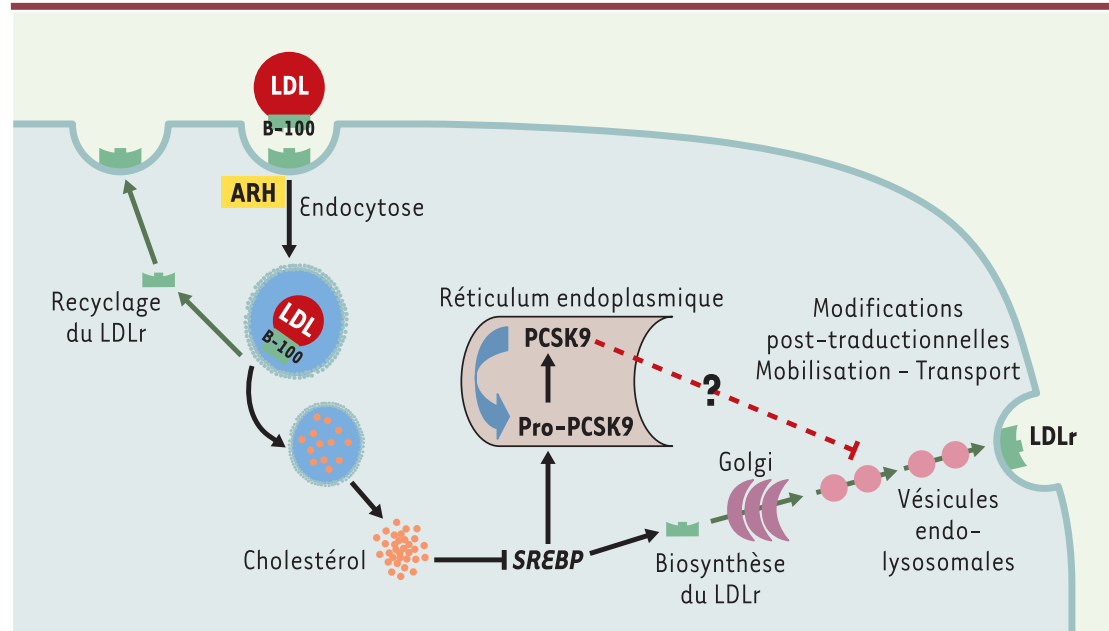

Figure 2. PCSK9 et métabolisme intracellulaire du récepteur des LDL. Le récepteur des LDL (LDLr) présent à la surface des hépatocytes en interagissant avec son ligand l'apoB100 capte les LDL plasmatiques. L'internalisation par endocytose des complexes LDL/LDLr au niveau de puits de clathrine nécessite l'interaction avec ARH. Le récepteur des LDL est recyclé et retourne à la membrane plasmique tandis que le cholestérol ainsi capté s'accumule dans l'hépatocyte. L'accumulation intracellulaire du cholestérol inhibe les facteurs de transcription SREBP qui, euxmêmes, activent l'expression du gène codant pour le LDLr. L'expression génique de PCSK9 est également activée par SREBP, favorisant ainsi la synthèse de pro-PCSK9. Après avoir clivé pro-PCSK9 en protéine mature dans le réticulum endoplasmique, PCSK9 inhibe de façon indirecte un ou plusieurs événements post-traductionnels ou la mobilisation du LDLr vers la membrane plasmique, vraisemblablement au niveau golgien ou endo/lysosomal. que par une diminution ou une abolition de son activité normale.

L'ensemble de ces études permet de conclure que PCSK9 joue un rôle antagoniste majeur sur l'expression et l'activité $\mathrm{du}$ récepteur des LDL in vivo. En revanche, les mécanismes moléculaires qui président à cette régulation restent à découvrir.

Un mécanisme d'action encore inconnu Dans la cellule, PCSK9 n'agit pas directement sur le récepteur des LDL [12]. Les voies métaboliques impliquant PCSK9 ne sont pas non plus altérées chez les souris déficientes en $A R H$, une protéine qui permet l'endocytose du récepteur des LDL [1]. Cela exclut la possibilité d'une action de PCSK9 pendant ou après l'étape d'internalisation du récepteur des LDL [14]. Par ailleurs, I'étude du mutant S127R suggère que l'action de PCSK9 sur le récepteur des LDL est un phénomène post-traductionnel tardif (Golgi, endosomes et/ou lysosomes) - probablement une augmentation de sa dégradation - conduisant à une diminution de l'expression du récepteur des LDL à la surface des cellules (Figure 2).

L'observation de l'action catalytique de PCSK9 sur pro-PCSK9 soulève de nouvelles interrogations. En effet, les substitutions D374y et S127R se traduisent par une diminution de la conversion dans le réticulum endoplasmique de pro-PCSK9 en PCSK9 mature. En revanche, la mutation F216L, la double mutation D374Y+N157K présentes sur le même allèle, ainsi que deux mutations identifiées très récemment, R218S et R237W, n'ont pas d'effet sur la maturation de pro-PCSK9. Or, toutes ces mutations affectent l'expression du récepteur des $\mathrm{LDL}$ et provoquent une hypercholestérolémie familiale. Enfin, de façon très inattendue, la mutation de la sérine active de PCSK9 (mutation S386A, non retrouvée chez les patients testés in vitro) qui se traduit par une absence totale de la forme mature de PCSK9 - proPCSK9 n'étant pas clivé - n'a pas d'effet sur l'expression du récepteur des LDL.

Ainsi, l'hypothèse la plus vraisemblable est 
que le clivage de pro-PCSK9 en PCSK9 est indispensable à son action sur le récepteur des LDL (le Golgi et/ou les endo/lysosomes seraient les sites d'action où pourrait s'opérer la régulation par PCSK9 de l'expression du récepteur des LDL). À partir des données actuelles, on ne peut cependant pas exclure que PCSK9 ait un autre mécanisme d'action qui reste à déterminer.

\section{PCSK9 et production de VLDL}

L'étude métabolique de l'apoB100 chez deux patients ayant une mutation S127R indique qu'à côté de la diminution du catabolisme hépatique, ces patients ont une production fortement augmentée de l'apoB100 des VLDL (very low density lipoproteins) (lipoprotéines de très basse densité, précurseurs des LDL) par rapport à celle de sujets témoins [13]. Cette observation n'est pas reproduite dans les modèles animaux ou cellulaires: la surexpression de la forme sauvage de PCSK9, chez la souris ou en culture cellulaire, ne provoque pas de modification de la production d'apoB100 et/ou de VLDL (G. Lambert, données non publiées). De même, la surexpression de PCSK9 sauvage ou muté (S127R) ne semble pas affecter la production de VLDL chez la souris déficiente en récepteur des LDL [14]. D’autres études sont nécessaires pour établir les méca- nismes cellulaires de cette nouvelle action de PCSK9 muté.

Ainsi, PCSK9 est bien un nouveau gène directement impliqué dans I'hypercholestérolémie familiale. Son action antagoniste sur l'expression et l'activité du récepteur des LDL est désormais établie avec certitude. L'étude réalisée par des techniques de pointe de biologie cellulaire ainsi que la production de souris dont le gène codant pour PCSK9 a été invalidé devraient permettre d'appréhender avec précision les mécanismes moléculaires présidés par PCSK9 et de proposer, éventuellement, une approche thérapeutique d'inhibition de cette voie métabolique. $\nabla$

PCSK9: a new gene involved in familial hypercholesteremia

\section{RéFÉRENCES}

1. Rader DJ, Cohen J, Hobbs HH. Monogenic hypercholesterolemia: New insights in pathogenesis and treatment. J Clin Invest 2003; 111: 1795-803.

2. Hunt SC, Hopkins PN, Bulka K, et al. Genetic localization to chromosome lp32 of the third locus for familial hypercholesterolemia in a Utah kindred. Arterioscler Thromb Vasc Biol 2000; 20: 1089-93.

3. Varret M, Rabes JP, Saint-Jore B, et al. Third major locus for autosomal dominant hypercholesterolemia maps to 1p34.1-p32. Am J Hum Genet 1999; 64: 1378-87.

4. Abifadel M, Varret M, Rabes JP, et al. Mutations in PCSK9 cause autosomal dominant hypercholesterolemia. Nat Genet 2003; 34: 154-6.
5. Timms KM, Wagner S, Samuels ME, et al. A mutation in PCSK9 causing autosomal-dominant

hypercholesterolemia in a Utah pedigree. Hum Genet 2004; 114: 349-53.

6. Leren TP. Mutations in the PCSK9 gene in Norwegian subjects with autosomal dominant

hypercholesterolemia. Clin Genet 2004; 65: 419-22.

7. Seidah NG, Benjannet S, Wickham L, et al. The secretory proprotein convertase neural apoptosisregulated convertase 1 (NARC-1): Liver regeneration and neuronal differentiation. Proc Natl Acad Sci USA 2003; 100: 928-33.

8. Maxwell KN, Soccio RE, Duncan EM, et al. Novel putative SREBP and LXR target genes identified by microarray analysis in liver of cholesterol-fed mice.J Lipid Res 2003; 44: 2109-19.

9. Horton JD, Shah NA, Warrington JA, et al. Combined analysis of oligonucleotide microarray data from transgenic and knockout mice identifies direct SREBP target genes. Proc Natl Acad Sci USA 2003; 100 : 12027-32

10. Dubuc G, Chamberland A, Wassef H, et al. Statins upregulate PCSK9, the gene encoding the proprotein convertase neural apoptosis-regulated convertase-1 implicated in familial hypercholesterolemia. Arterioscler Thromb Vasc Biol 2004; 24: 1-6.

11. Maxwell KN, Breslow JL. Adenoviral-mediated expression of Pcsk9 in mice results in a low-density lipoprotein receptor knockout phenotype. Proc Natl Acad Sci USA 2004; 101: 7100-5.

12. Benjannet $S$, Rhainds $D$, Essalmani R, et al. NARC1/PCSK9 and its natural mutants: zymogen cleavage and effects on the LDLR and LDL-cholesterol. J Biol Chem 2004 (sous presse).

13. Ouguerram K, Chetiveaux M, Zair Y, et al. Apolipoprotein B100 metabolism in autosomaldominant hypercholesterolemia related to mutations in PCSK9. Arterioscler Thromb Vasc Biol 2004; 24: 1448-53.

14. Park SW, Moon YA, Horton JD. Post-transcriptional regulation of $L D L$ receptor protein by proprotein convertase subtilisin/kexin type 9a (PCSK9) in mouse liver.J Biol Chem 2004 (sous presse).

\section{NOUVELLE}

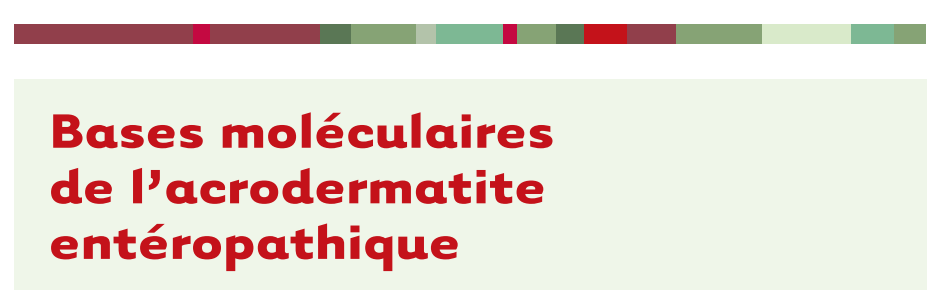

Sébastien Küry, Stéphane Bézieau, Jean-Paul Moisan

> L'acrodermatite entéropathique $(A \varepsilon)$ est un syndrome héréditaire rare de déficience en zinc, transmis selon un mode autosomique récessif. Apparaissant dès la naissance ou au moment du sevrage [1], l'AE se définit théoriquement par la présence de trois symptômes pathogno- moniques: une dermatite acrale (des extrémités) et péri-orificielle, une diarrhée et une alopécie [2]. Ces symptômes sont la conséquence précoce d'une déficience nutritionnelle en zinc due à une malabsorption dans le duodénum et le jéjunum. Le corps humain ne possédant
S. Küry, J.P. Moisan:

Laboratoire d'étude du

polymorphisme de l'ADN, Faculté de Médecine, 1 , rue Gaston Veil, 44035 Nantes Cedex, France.

S. Béziau: Laboratoire d'étude du polymorphisme de l'ADN et Service de Génétique médicale, $\mathrm{CHU}$ de Nantes, Hôtel Dieu, 44093 Nantes Cedex 1, France.

skury@sante.univ-nantes.fr

pas de véritable réserve de zinc, la déficience s'étend rapidement à tout l'organisme et se manifeste par un tableau cli- 